\title{
A New Automated Way to Measure Polyethylene Wear in THA Using a High Resolution CT Scanner: Method and Analysis
}

\author{
Gerald Q. Maguire Jr., ${ }^{1}$ Marilyn E. Noz, ${ }^{2}$ Henrik Olivecrona, ${ }^{3}$ \\ Michael P. Zeleznik, ${ }^{4}$ and Lars Weidenhielm ${ }^{3}$ \\ ${ }^{1}$ School of Information and Communication Technology, KTH Royal Institute of Technology, Isafjordsgatan 26, \\ 418 164-40 Stockholm, Sweden \\ ${ }^{2}$ Department of Radiology, New York University, 550 First Avenue TSHW232, New York, NY 10016, USA \\ ${ }^{3}$ Department of Molecular Medicine and Surgery, Section of Orthopaedics and Sports Medicine, Karolinska Institute, \\ A2:07, 17176 Stockholm, Sweden \\ ${ }^{4}$ School of Computing, University of Utah, College of Engineering, 50 Central Campus Dr., Room 3190, Salt Lake City, UT 84112, USA \\ Correspondence should be addressed to Marilyn E. Noz; marilyne.noz@gmail.com
}

Received 4 October 2013; Accepted 7 November 2013; Published 22 January 2014

Academic Editors: J. U. Carmona and Y. K. Tu

Copyright ( 2014 Gerald Q. Maguire Jr. et al. This is an open access article distributed under the Creative Commons Attribution License, which permits unrestricted use, distribution, and reproduction in any medium, provided the original work is properly cited.

\begin{abstract}
As the most advantageous total hip arthroplasty (THA) operation is the first, timely replacement of only the liner is socially and economically important because the utilization of THA is increasing as younger and more active patients are receiving implants and they are living longer. Automatic algorithms were developed to infer liner wear by estimating the separation between the acetabular cup and femoral component head given a computed tomography (CT) volume. Two series of CT volumes of a hip phantom were acquired with the femoral component head placed at 14 different positions relative to the acetabular cup. The mean and standard deviation (SD) of the diameter of the acetabular cup and femoral component head, in addition to the range of error in the expected wear values and the repeatability of all the measurements, were calculated. The algorithms resulted in a mean $( \pm S D)$ for the diameter of the acetabular cup of $54.21( \pm 0.011) \mathrm{mm}$ and for the femoral component head of $22.09( \pm 0.02) \mathrm{mm}$. The wear error was $\pm 0.1 \mathrm{~mm}$ and the repeatability was $0.077 \mathrm{~mm}$. This approach is applicable clinically as it utilizes readily available computed tomography imaging systems and requires only five minutes of human interaction.
\end{abstract}

\section{Introduction}

Total hip arthroplasty (THA) devices are being utilized for longer periods of time as younger and more active patients receive them [1]. Although there are a variety of common reasons for long-term failure [2,3], this study concerns only wear [4]. In vivo wear rates of several different acetabular cups, with and without polyethylene liners, have been reported [5-13] with the most recent liner wear rates ranging from $0.037 \mathrm{~mm} /$ year to $0.005 \mathrm{~mm} /$ year and total wear at revision being about 1.0 to $3.5 \mathrm{~mm}$. Higher precision and accuracy of wear assessment methods would shorten the time for clinical studies of new implants and enable detection of clinically significant wear [14].

Previously our group showed that CT volumes can be used to evaluate acetabular cup position and migration in hip phantoms and patients $[15,16]$ and to determine $3 \mathrm{D}$ migration of the femoral component head into the acetabular cup at $1 \mathrm{~mm}$, later reduced to $0.51 \mathrm{~mm}$ [17]. However, this required considerable user interaction time as about 200 landmarks (points) had to be placed on the 3D surfaces of the femoral component head and acetabular cup.

Here the interaction time to choose landmarks is reduced by limiting the number of landmarks to a total of seven (requiring only five minutes on average per CT volume) and the skill level of the operator was reduced. Surfaces were automatically extracted based on these landmarks plus a threshold for the electron density of the prosthetic material; the center and diameter of the prosthetic components viewed as (parts of) spheres were automatically determined, and from these centers, the distance between the acetabular cup and femoral 
TABLE 1: Phantom displacement and distance difference as determined from the micrometer settings.

\begin{tabular}{|c|c|c|c|c|c|}
\hline \multicolumn{2}{|c|}{$\begin{array}{l}\text { Scan series identifier (letters identify scan } \\
1 \text { to } 14 \text { in each series) }\end{array}$} & \multicolumn{3}{|c|}{ Displacement (mm) } & \multirow{2}{*}{$\begin{array}{l}\text { Expected CupHeadSeparation }(\mathrm{mm}) \text { distance between } \\
\text { acetabular cup and femoral component head centers } \\
\text { relative to the reference scans (T, A) with a starting } \\
\text { position of } 0.00 \mathrm{~mm}\end{array}$} \\
\hline One & Two & $X$ & $Y$ & $Z$ & \\
\hline $\mathrm{T}$ & A & 0.000 & 0.000 & 0.000 & 0.000 \\
\hline $\mathrm{K}$ & G & 0.000 & 0.000 & 0.000 & 0.000 \\
\hline $\mathrm{X}$ & $\mathrm{T}$ & 0.050 & 0.050 & 0.050 & 0.087 \\
\hline $\mathrm{W}$ & $\mathrm{F}$ & 0.100 & 0.100 & 0.100 & 0.173 \\
\hline $\mathrm{N}$ & $\mathrm{R}$ & 0.150 & 0.100 & 0.100 & 0.206 \\
\hline $\mathrm{P}$ & M & 0.150 & 0.150 & 0.100 & 0.235 \\
\hline $\mathrm{A}$ & $\mathrm{Y}$ & 0.150 & 0.150 & 0.150 & 0.260 \\
\hline $\mathrm{R}$ & $\mathrm{D}$ & 0.200 & 0.200 & 0.200 & 0.346 \\
\hline $\mathrm{H}$ & W & 0.250 & 0.250 & 0.250 & 0.433 \\
\hline $\mathrm{Z}$ & $\mathrm{C}$ & 0.300 & 0.300 & 0.300 & 0.520 \\
\hline $\mathrm{D}$ & K & 0.350 & 0.350 & 0.350 & 0.606 \\
\hline M & $\mathrm{P}$ & 0.400 & 0.400 & 0.400 & 0.692 \\
\hline $\mathrm{E}$ & $\mathrm{V}$ & 0.450 & 0.450 & 0.450 & 0.779 \\
\hline S & Z & 0.500 & 0.500 & 0.500 & 0.866 \\
\hline
\end{tabular}

component head is inferred. The entire process takes roughly 20 minutes.

\section{Methods and Materials}

A hip phantom, used to simulate the 3D displacement of the femoral component (CoCr PROTASUL-20, Zimmer Inc., Warsaw, ID) toward the acetabular cup (Inter-OpTM, Zimmer Inc., Warsaw, ID, formally Sulzer Orthopaedics), was scanned in a high resolution prototype flat panel CT scanner (Siemens Medical Solutions, Erlangen, Germany) [18]. The same experimenter acquired two series of 14 scans separated by five days. Before each scan, the hip phantom was removed from the CT scanner, the micrometer was set as indicated in Table 1, and then the hip phantom was replaced with the femur roughly aligned with the $z$-axis of the scanner. Each scan was identified by a random letter. The CT scan parameters were $100 \mathrm{kVp}$ and $50 \mathrm{~mA}$, with a spatial resolution of $200 \mu \mathrm{m}$ on a side, a matrix size of $512 \times 512$, and 390 slices for the first and 385 slices for the second scan.

The phantom was disassembled and the diameter of the acetabular cup was measured using a coordinate measuring machine (CMM) (Global Advantage, Brown and Sharp, North Kingstown, RI) with special software (PC-DMIS, Version 1.0, Wilcox Associates, Inc., North Kingstown, RI) by an outside vendor (Hexagon Metrology, Inc., North Kingstown,
RI). The ruby probe tip had a diameter of $3 \mathrm{~mm}$ on a $20 \mathrm{~mm}$ shaft. A total of 8 probe positions (each with an accuracy of $0.000254 \mathrm{~mm}$ and an assumed repeatability of $0.000508 \mathrm{~mm}$ ) were used. The diameter of the femoral component head was measured using a caliper (Mitutoyo Corporation Digimatic, Toronto, ON, Canada), calibrated by the manufacturer. The same experimenter made 10 measurements moving the caliper to a different position each time.

A 3D image processing tool, previously described in [19, 20], was used to choose seven landmarks, readily identifiable in a patient. These landmarks were used only to limit the CT data volume searched to find the surfaces of the acetabular cup and the femoral component head. Details of the hardware and software are given in the appendix.

2.1. Procedure for Choosing Landmarks and Algorithm for Automatic Surface Extraction. (A) Viewing each CT volume as a $3 \mathrm{D}$ isosurface, the observer placed a landmark on three noncolinear points on the bottom of the acetabular cup to define a plane. A fourth landmark was placed near the apex (apical dome hole). Viewing the CT volume as 2D orthogonal projections: transverse, coronal, and sagittal (Figure 1), the observer centered a suitably sized 3D sphere in the apical dome hole and on the coronal projection, shifted it diagonally out a short distance ( 5 to $10 \mathrm{~mm}$ ), and created a landmark (Figure 2). 


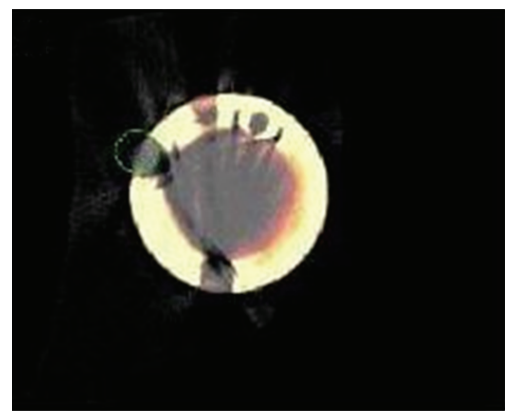

(a)

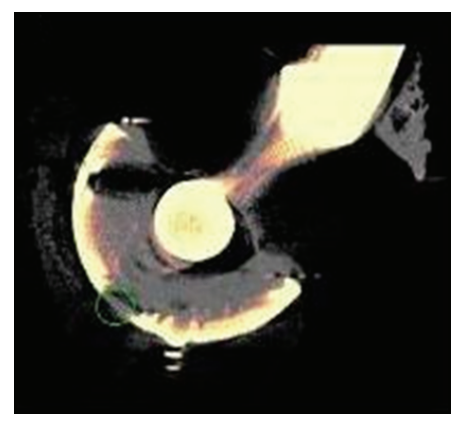

(b)

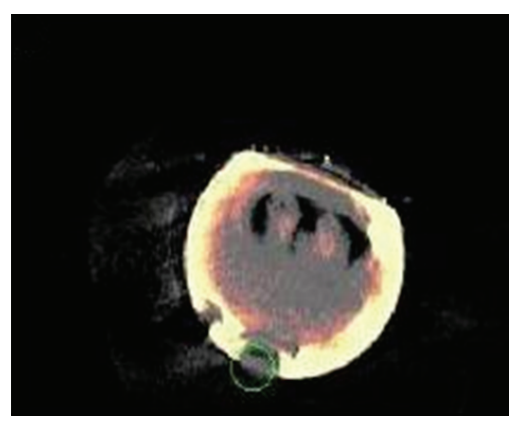

(c)

FIGURE 1: Three 2D orthogonal projections showing the 3D spherical landmark located in the apical dome hole. (a) shows the axial projection, (b) the coronal, and (c) the sagittal.

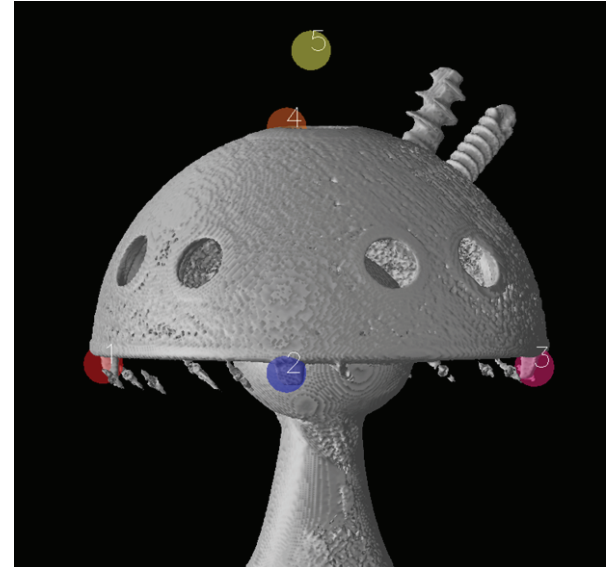

FIgURE 2: The five landmarks chosen on the acetabular cup are shown in 3D. Only landmarks 1, 2, 3, and 5 are used in the surface extractor calculations. Landmark 4 is used only by the operator to locate the apical dome hole.

(B) A surface extraction algorithm used these landmarks and a threshold (based on the acetabular cup material and the peak kilovoltage) to limit the search. The shortest distance between the defined plane and the point centered in the apical dome hole defined a starting radius. The software searched from the outside and ranged for only $60 \%$ of the starting radius to eliminate unused screw holes.

(C) Using 2D orthogonal projections the observer interactively placed a $3 \mathrm{D}$ sphere so as to surround the junction between the femoral component head and stem and placed a landmark. A new 3D sphere was then placed which encompassed the femoral component head and a landmark was placed. This landmark together with the stem landmark constrained the search for the surface from extending into the stem (Figure 3).

(D) The surface extraction algorithm searched from the outside of the femoral component head inward and ended when the voxel value no longer exceeded the threshold, as femoral component heads are not necessarily composed of solid metal.

(E) For either surface, the algorithm found points where the voxel values along the search path consistently exceeded the threshold so that isolated voxels (above threshold) are ignored.

2.2. Algorithm for Automatic Sphere Fitting to the Extracted Surfaces. The surface extraction algorithm produced a set of initial landmarks (2,000-16,000) for each surface and the sphere fitting algorithm used these to estimate the actual center of the hemisphere or sphere (as appropriate) and to compute the diameter as these landmarks must statistically be consistent with a given center and diameter. The sphere fitting algorithm selected 80,000 random sets $(x, y, z)$ of four surface landmarks (with every pair separated by at least $2 \mathrm{~mm}$ to avoid degenerate cases) to estimate a radius and center. The first time, a 1000 bin histogram of possible integer radii was computed and the maximum bin defined the initial radius value. Next, this procedure was repeated and statistics were kept per landmark of how many times that landmark contributed to an estimate of a radius that exceeded the initial radius value by $\pm 2 \mathrm{~mm}$. Landmarks with high values (greater than 1,600$)$ were removed. This was repeated again and statistics were kept per landmark of how many times the landmark contributed to the estimate of a radius that exceeded the initial radius value by twice the slice thickness $( \pm 0.4 \mathrm{~mm})$. Typically the initial set of landmarks, approximately 15,000 for the acetabular cup and 2,000 landmarks for the femoral component head, was reduced to $12,500-13,000$ and $950-1,100$, respectively.

2.3. Observer Interaction. The initial seven landmarks were chosen by the same observer (who was not the experimenter and had no knowledge of the scan order) on scan series one on one day and again nine days later. The observer chose landmarks on scan series two one day after those for scan series one and again twelve days later.

2.4. Analysis. For each scan, after landmarks selection, the surface extraction algorithm and the sphere fitting algorithm were applied. The initial and final landmarks were visually verified for correct placement on the intended surface. The calculated separation (CupHeadSeparation) was simply the distance between the defined sphere centers. All data, including the scan identifier, initial radius and center $(x, y, z)$, and 

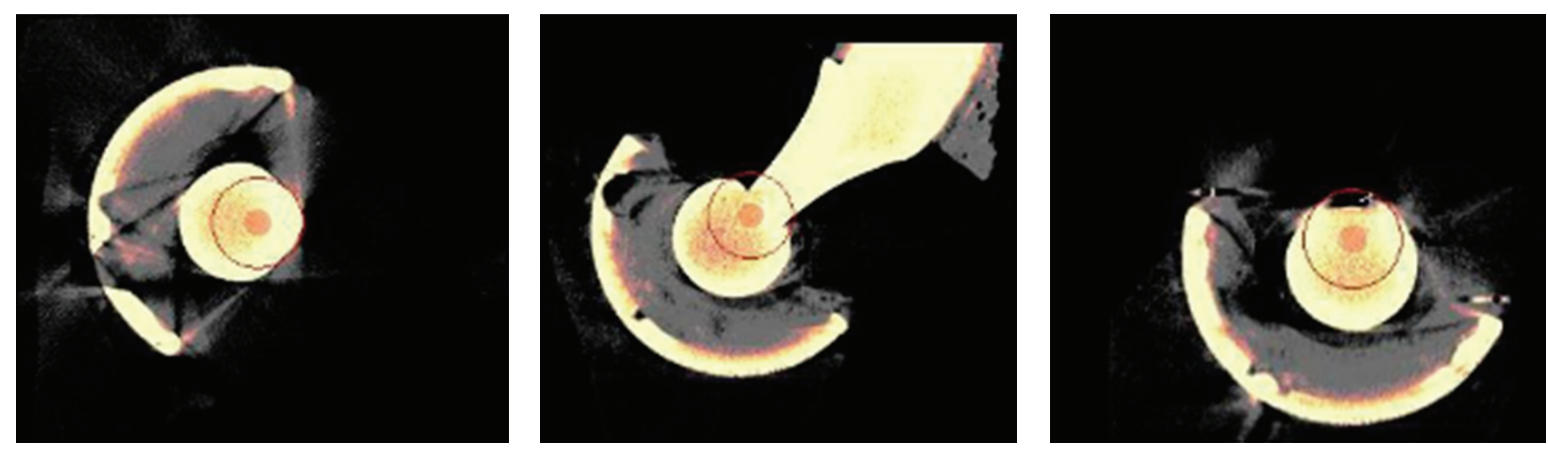

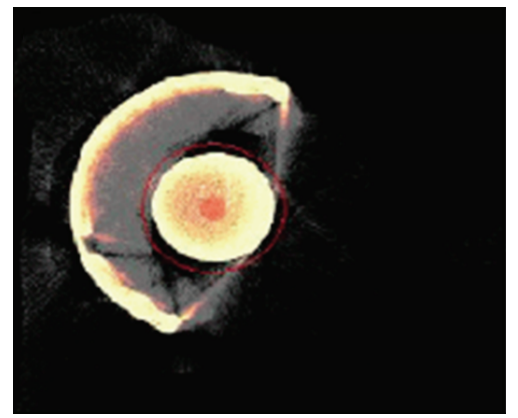

(a)

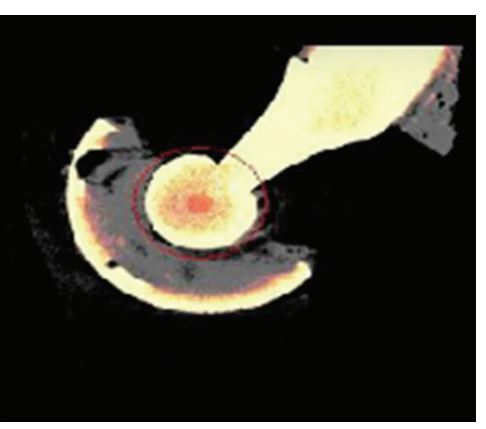

(b)

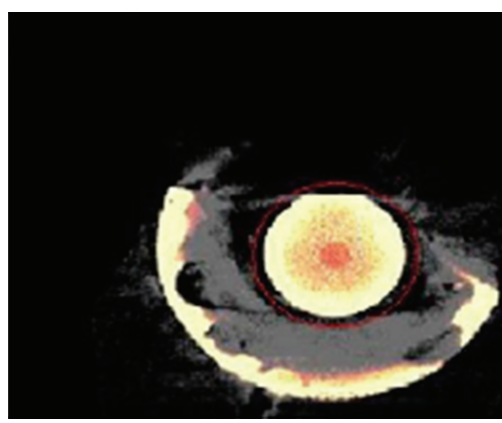

(c)

FIGURE 3: The top row contains the three 2D orthogonal projections showing the 3D spherical landmark located at the junction between the femoral component head and stem and the bottom row contains the three $2 \mathrm{D}$ orthogonal projections showing the 3D spherical landmark surrounding the femoral component head. (a) shows the axial projection, (b) the coronal, and (c) the sagittal.

TABLE 2: Acetabular cup and head diameter measurements (the mean and median were quite close) based on experimental results and physical measurements.

\begin{tabular}{|c|c|c|c|c|c|}
\hline \multirow{2}{*}{ Component } & \multirow{2}{*}{$\begin{array}{c}\text { Diameter }(\mathrm{mm}) \\
\text { Mean }( \pm \mathrm{SD})\end{array}$} & \multirow{2}{*}{ Coefficient of variation } & \multicolumn{2}{|c|}{ 99\% Confidence interval (mm) } & \multirow{2}{*}{ Measured (mm) } \\
\hline & & & Lower & Upper & \\
\hline Acetabular cup & $54.22( \pm 0.011)$ & 0.024 & 54.226 & 54.234 & $54.855^{\mathrm{a}}$ \\
\hline Femoral head & $22.09( \pm 0.02)$ & 0.14 & 22.09 & 22.11 & $22.10( \pm 0.01)^{\mathrm{b}}$ \\
\hline
\end{tabular}

${ }^{\mathrm{a} C C M},{ }^{\mathrm{b}}$ Calipers.

the final (best) radius and center $(x, y, z)$ all in millimeters, were saved in a file as a comma separated list (CSV).

2.5. Statistical Analysis. For each trial, the sphere diameters were tabulated, resulting in a total of 56 data points ( 2 trials $*$ 14 scans $* 2$ series) for the acetabular cup and for the femoral component head. These diameter data were tested graphically for normality (histogram, box, density, and quantile-quantile plots) and then the median, mean, standard deviation (SD), and coefficient of variance were calculated, as well as the 99\% confidence interval (CI). For each trial of each scan, the CupHeadSeparation was calculated and the difference from the expected value based on the micrometer settings (Table 1) was calculated and plotted. The repeatability of the test results was evaluated as outlined in $[21,22]$ as well as the $99 \%$ CI. The repeatability was defined as the precision under conditions where independent tests are conducted with the same method on the same test item in the same laboratory by the same observer using the same equipment, within a short interval of time. $\mathrm{R}$ version 2.11.1 was used for all statistical calculations.

\section{Results}

All scans in both series were able to be analyzed for both trials. The visual check of the generated surface landmarks after surface extraction (Figure 4(a)) and again after sphere fitting (Figure 4(b)) confirmed that the landmarks were correctly placed for the acetabular cup (top row) and the femoral component head (bottom row).

The calculated diameter for both the acetabular cup and the femoral component head was graphically checked to confirm that the data were normally distributed. The diameter mean and median values, which were almost equal, for the acetabular cup and femoral component as well the coefficient of variance, the $99 \%$ confidence interval, and values obtained by the CMM and caliper measurements are given in Table 2. Figure 5 illustrates the acetabular cup data graphically and Figure 6 does the same for the femoral component head.

For each trial of each scan, the CupHeadSeparation was calculated along with the difference between this and the expected CupHeadSeparation based on the micrometer settings (Table 1). The reference scan was the first scan in which 

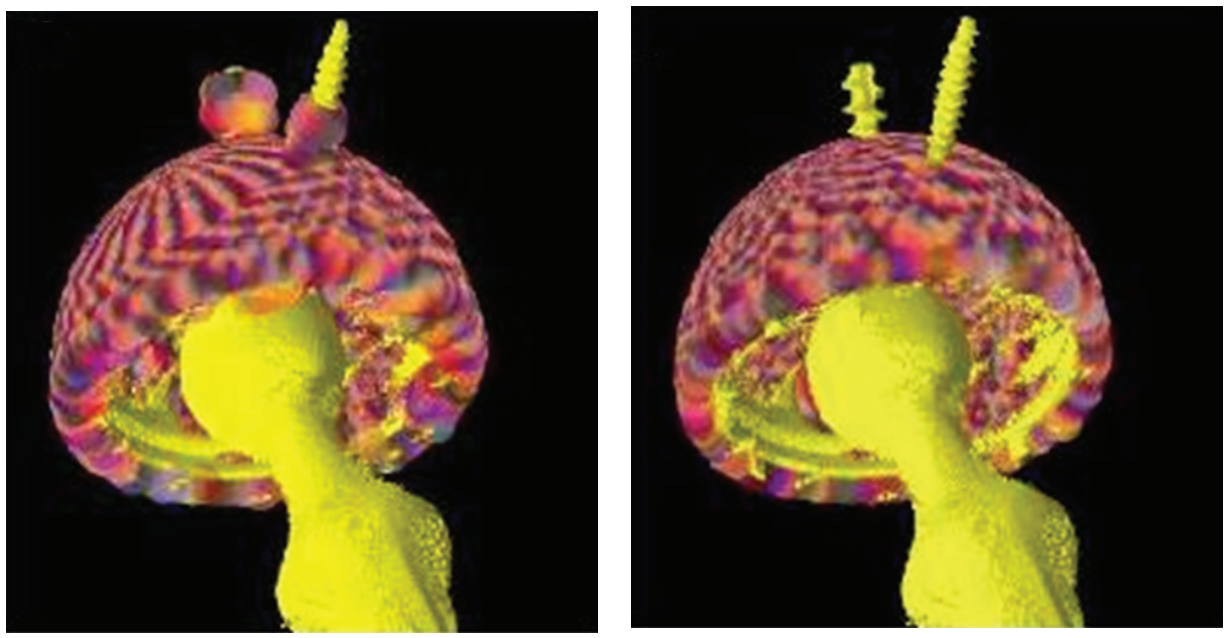

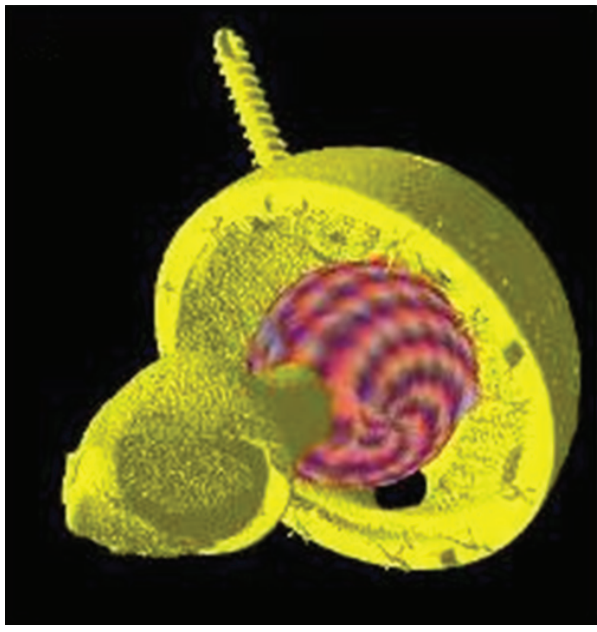

(a)

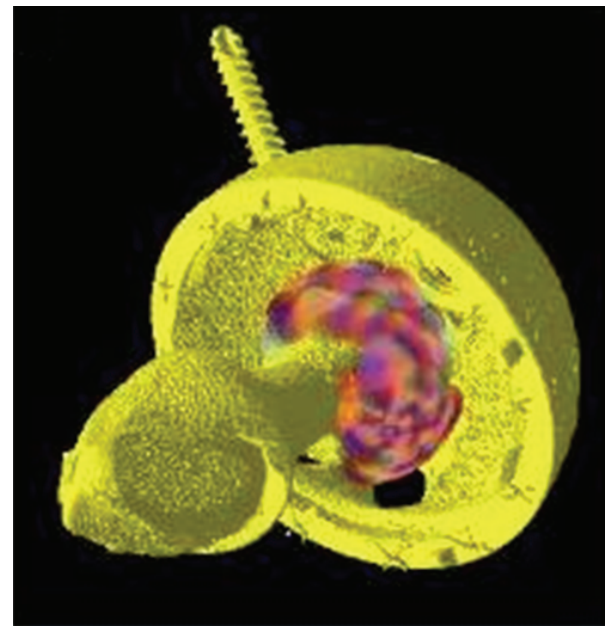

(b)

FIGURE 4: The top row illustrates the initial and valid surface landmarks produced on the acetabular cup by the surface extraction algorithm and the bottom row shows those for the femoral component head. The set of initial landmarks produced is shown in (a). The set of valid landmarks is shown in (b). The overlapping colored spherical glyphs represent the generated landmarks.

each micrometer was set to zero. For both scan series and trials, the linear least squares line fit between the measured and expected values was quite close to a $45^{\circ}$ line; that is, the measured and expected values were approximately equal (Figure 7). The residuals of the regression, the line intercept, and the slope values are summarized in Table 3. As can be seen from Figure 7, all the distance results fell within the interval of $\pm 0.1 \mathrm{~mm}$ with a mean of $-0.013 \mathrm{~mm}$, a SD of $\pm 0.038 \mathrm{~mm}$, and a $99 \%$ CI of -0.031 to $0.006 \mathrm{~mm}$. Repeatability was $0.077 \mathrm{~mm}$ for the 224 (14 scans $* 4$ (radius + center points) $* 2$ trials $* 2$ series) values. The mean and median of the difference in each value were $0.004 \mathrm{~mm}$ and $0.003 \mathrm{~mm}$, respectively, with a standard deviation of $\pm 0.028 \mathrm{~mm}$ and a $99 \%$ CI of 0.009 to $-0.0004 \mathrm{~mm}$.

\section{Discussion}

Existing wear measurement techniques range from simple single radiographic techniques to more advanced threedimensional (3D) techniques [4, 5, 23-30]. The most accurate
TABLE 3: Linear regression analysis results based on $45^{\circ}$ line for experimentally determined distance versus expected measurements.

\begin{tabular}{lccc}
\hline & Value & Standard error & $P$ value \\
\hline Regression residuals & $3208.22(F$-value $)$ & 0.02 & 0 \\
Line intercept & 0.02 & 0.01 & 0.01 \\
Slope & 0.95 & 0.02 & 0 \\
\hline
\end{tabular}

$3 \mathrm{D}$ wear monitoring method today is radiostereometric analysis (RSA) [31, 32]. However, RSA is normally not available in clinical practice since it requires special stereotactic X-ray equipment and implantation of small tantalum balls. For 3D wear assessment current multislice computed tomography (CT) offers accurate spatial volume resolution without significant metal artefacts, now well suppressed by the CT manufacturers' software. A measurement of femoral component head penetration into polyethylene using a 3D CT technique was reported in [23]. 


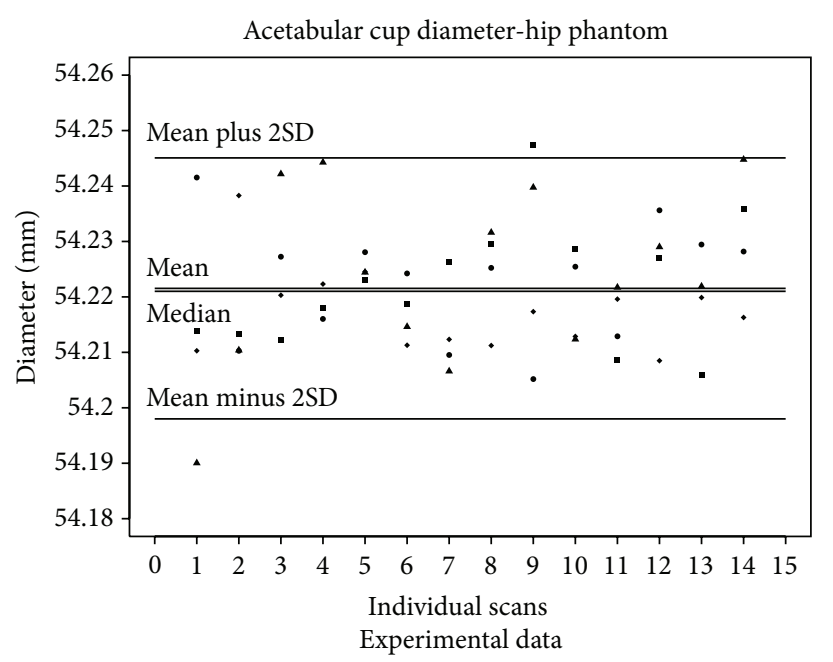

- Scan series one-trial one

- Scan series one-trial two

- Scan series two-trial one

- Scan series two-trial two

FIGURE 5: The values of the acetabular cup diameters obtained from each individual calculation are shown with the median, mean, and two SD lines drawn through the data. The median value is quite close to the mean value.

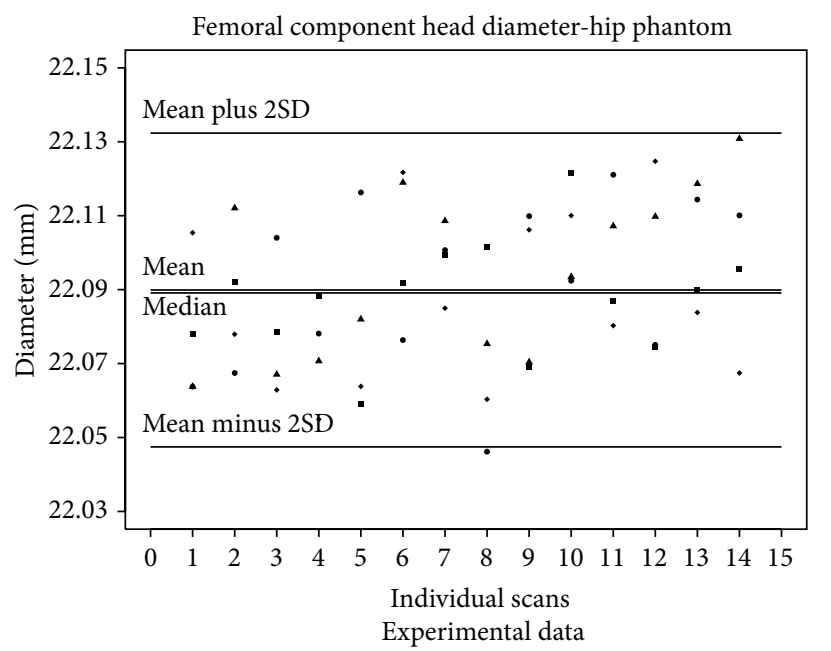

- Scan series one-trial one

- Scan series one-trial two

- Scan series two-trial one

- Scan series two-trial two

FIgURE 6: The values of the femoral component head diameters obtained from each individual calculation are shown with the median, mean, and two SD lines drawn through the data. The median value is quite close to the mean value.

The present study reports a method to assess polyethylene wear by measuring the displacement of the femoral component head relative to the acetabular cup, thus giving a baseline for an ideal situation. In a clinical situation, there is the possibility that the femoral component head is not seated at the most worn part of the polyethylene, especially in the presence of multiple wear tracts as demonstrated in our previous study [33]; there is a risk of distortion of the volume due to patient movement during the scan, and finally, the proposed

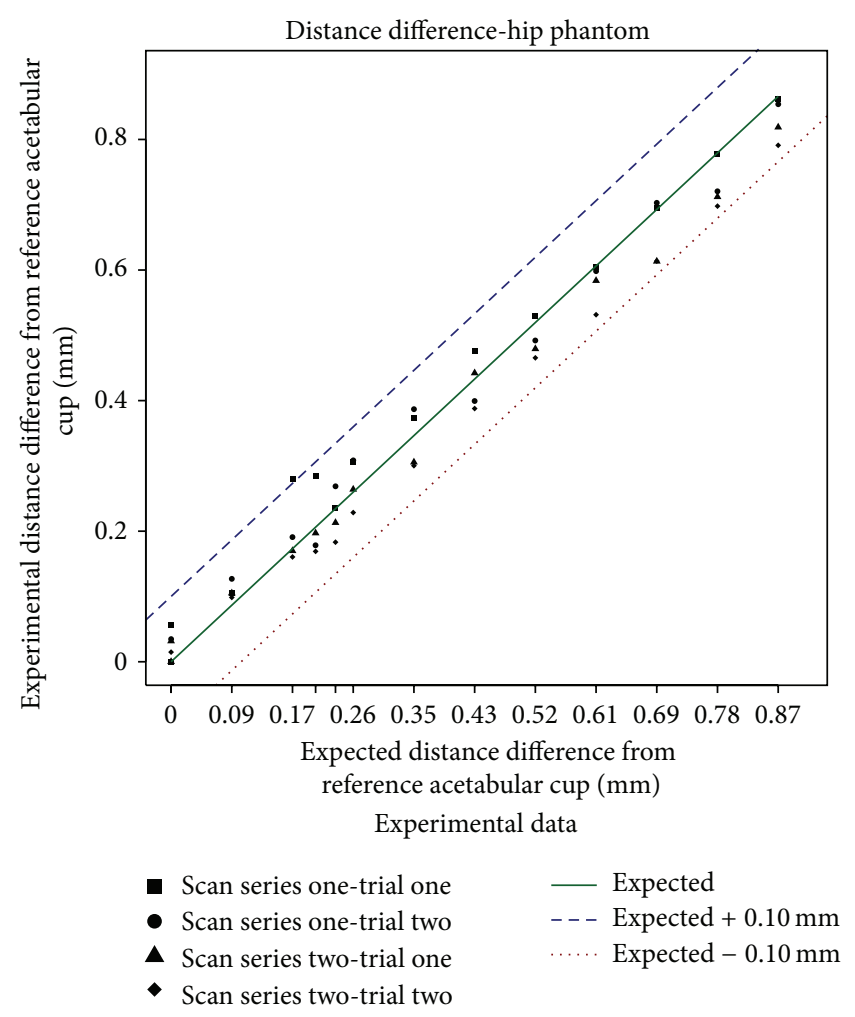

Figure 7: The calculated CupHeadSeparation plotted against the expected CupHeadSeparation from Table 1, for scan series one and two (both trials). Lines are drawn at the exact expected value and at $\pm 0.1 \mathrm{~mm}$ demonstrating the data were within $0.1 \mathrm{~mm}$ of the expected distance.

method was devised for uncemented acetabular components of hemispherical configuration which comprise the majority of acetabular cups in clinical use today. The agreement of $\pm 0.1 \mathrm{~mm}$ between the calculated CupHeadSeparation and the expected CupHeadSeparation was reasonably good for both scan series and suggests that this method could be used clinially to detect differences of greater than $0.1 \mathrm{~mm}$. The centers of the acetabular cup and the femoral component head are implicitly known to be within $\pm 1 \mathrm{~mm}$ ( $\mathrm{SD} \pm 0.013 \mathrm{~mm}$ and $\pm 0.03 \mathrm{~mm}$, resp.). Hence the distance between the centers should be within $\pm 0.086 \mathrm{~mm}$ of the actual distance. This improves the previous results from our group [17] but is about half the accuracy of phantom study results presented in [23]. However, this new method does not need special reconstruction of the raw CT data and the computer aided design (and manufacturing) data for the actebular cup and femoral component, nor does it need 40 points on the femoral component head and 20 or more around the bottom rim of the acetabular cup. The software for the method presented is system independent whereas [23] uses the software package OSIRIS [34] which runs only on an Apple computer. The accuracy and repeatability in [23] were determined by repeated measurements on a calibrated $28 \mathrm{~mm}$ prosthetic head and by comparing them with direct metrological measurements on acetabular specimens with in vitro wear from machining and 
on explanted acetabular specimens with in vivo wear. Estimated femoral component penetration in both all-poly and metal-backed acetabular components ranged from 0.009 to $0.245 \mathrm{~mm}$ with a mean of $0.080 \mathrm{~mm}$ and SD $0.067 \mathrm{~mm}$ [23].

The calculated acetabular cup diameter measurements in this study were approximately $0.6 \mathrm{~mm}$ less than that obtained by CMM because the CMM probe tip ( $3 \mathrm{~mm}$ ) was too large to penetrate the projections of the rough trabecular metal mesh surface of the acetabular cup, resulting in a larger diameter defined by the highest projections of this surface. However, our sphere fitting function utilizes CT voxels representing the full thickness of the metal surface without the $0.3 \mathrm{~mm}$ thick trabecular coating. The estimated CT measurements of the femoral component head diameter were very close to the caliper measurement. These diameter measurements provide an indirect indication of the accuracy and repeatability of finding these surfaces. An additional aspect of this method is that with the addition of one more landmark on the acetabular cup there is sufficient data to register the same acetabular cup in two different $\mathrm{CT}$ volumes taken at different times.

The particular implant used here was discontinued due to a manufacturing error and the CT scanner was a prototype. Therefore, after the experiments described above we repeated the setup using a presently available acetabular cup (Cluster Holed Trilogy cup, diffusion bonded to a Tivanium TI-6AL$4 \mathrm{~V}$ alloy, Zimmer Inc., Warsaw, ID) with a nominal diameter of $56 \mathrm{~mm}$. The femoral component (Zimmer Inc., Warsaw, ID) had a nominal head diameter of $32 \mathrm{~mm}$. We scanned this phantom on a commercially available clinical CT scanner (SOMATOM Definition Flash, Siemens Medical Solutions, Forcheim, Germany) which has an $x-y$ pixel size of $0.35 \mathrm{~mm}$ and a slice thickness of $0.6 \mathrm{~mm}$. The volumes for this scan series were acquired with a matrix size of $512 \times 512$ for 357 slices. Preliminary analysis gave a mean (SD) for acetabular cup diameter and femoral component head, respectively, of $54.85( \pm 0.03) \mathrm{mm}$ and $32.23( \pm 0.07) \mathrm{mm}$. As described by Goldvasser et al. [35], the method has been shown to accurately estimate the displacement of the acetabular cup diameter and femoral component head: $x$-axis $0 \mathrm{~mm}$ (SD 0.213), $y$ axis $0.039 \mathrm{~mm}$ (SD 0.035), and $z$-axis $0.039 \mathrm{~mm}$ (SD 0.051). Although it was possible to resolve the CupHeadSeparation to $\pm 0.1 \mathrm{~mm}$, this needs further confirmation. We are currently calculating the liner wear from preoperative CT scans of patients who had their prosthesis explanted. These results are being compared to those obtained from direct measurement of the liner using calibers and CMM. On 11 samples thus far the results are consistent with those presented here. Future work will investigate simplification of this method as clinically, only the distance between the centers need be determined. Hence it should be possible to reduce the number of landmarks from seven to three: one to indicate where the stem of the femoral component joins the head, one spherical landmark that encloses the femoral component head, and one spherical landmark that encloses the acetabular cup. In this approach the surface of the femoral component head would first be found (establishing its radius and center), then the algorithm could search outward to find the outer acetabular cup surface. The third spherical landmark would limit this search. The outer surface of the acetabular cup would then be used to find the center of the acetabular cup, hence the distance between the centers can be computed. Note that this method would not be suitable if the femoral component is in direct contact with the acetabular cup.

\section{Conclusions}

In this paper we presented a potentially clinically applicable method to determine polyethylene wear associated with THA. This is important because younger and more active people are receiving these implants and they are living longer. As the first operation is the most successful one, if the liner only could be replaced, the patient would be spared a more extensive operation. The ability to replace the liner depends on early detection of liner wear. This method utilizing widely available CT machines brings us closer to this goal of timely detection of liner wear.

\section{Appendix}

\section{Hardware and Software Specifications}

The programs to calculate the sphere diameters and centers were written completely in $\mathrm{C}$ with no special libraries other than the libc and libm required and are compiled under standard GCC. The programs run on any standard Linux/UNIX configuration (we tested on SuSE 10.3, 11.x, and 12.x) and any standard PC (we tested on two different computers equipped with an Intel Xenon CPU one at $3.60 \mathrm{GHz}$ and the other at $2.80 \mathrm{GHz}$ (with the software and OS in 32 bit mode on both machines), two different Intel Pentium 4 CPUs each at 3.0 GHz (in 32 bit mode), an Intel Core 2 Duo CPU E6850 at $3.0 \mathrm{GHz}$ (64 bit mode), and an Intel Core 2 Quad CPU Q9550 at $2.83 \mathrm{GHz}$ (in 64 bit mode), an Intel Pentium D CPU at $2.80 \mathrm{GHz}$ (in 64 bit mode), and an Intel i7 $950 \mathrm{CPU}$ at $3.07 \mathrm{GHz}$ ) with configurations of $4-16 \mathrm{~GB}$ of memory. The running time for the extraction of the acetabular cup and femoral component head surface was 3-5 seconds, whereas the time for calculating the sphere center is 2 to 7 minutes, depending on the memory available and the speed of the CPU. The code used only a single processor; that is, it was single threaded code.

\section{Conflict of Interests}

The authors report no conflict of interests.

\section{Acknowledgments}

The authors would like to express their gratitude to Dr. Erica De Bernardo for help with statistics and to Dov Goldvasser for collecting the scan series.

\section{References}

[1] J. A. Singh, "Epidemiology of knee and hip arthroplasty: a systematic review," The Open Orthopaedics Journal, vol. 5, pp. 8085, 2011. 
[2] J. Karrholm, W. Frech, K.-G. Nilsson, and F. Snorrason, "Increased metal release from cemented femoral components made of titanium alloy: 19 hip prostheses followed with radiostereometry (RSA)," Acta Orthopaedica Scandinavica, vol. 65, no. 6, pp. 599-604, 1994.

[3] W. H. Harris, "Osteolysis and particle disease in hip replacement: a review," Acta Orthop Scand, vol. 65, no. 1, pp. 113-123, 1994.

[4] G. E. R. Thomas, D. J. Simpson, S. Mehmood et al., "The sevenyear wear of highly cross-linked polyethylene in total hip arthroplasty: a double-blind, randomized controlled trial using radiostereometric analysis," The Journal of Bone and Joint Surgery A, vol. 93, no. 8, pp. 716-722, 2011.

[5] X. Dai, H. Omori, Y. Okumura et al., "Serial measurement of polyethylene wear of well-fixed cementless metal-backed acetabular component in total hip arthroplasty: An over 10 year follow-up study," Artificial Organs, vol. 24, no. 9, pp. 746-751, 2000.

[6] D. F. Amanatullah, J. Landa, E. J. Strauss, J. P. Garino, S. H. Kim, and P. E. Di Cesare, "Comparison of surgical outcomes and implant wear between ceramic-ceramic and ceramicpolyethylene articulations in total hip arthroplasty," Journal of Arthroplasty, vol. 26, no. 6, pp. 72-77, 2011.

[7] M. Krismer, B. Stöckl, M. Fischer, R. Bauer, P. Mayrhofer, and M. Ogon, "Early migration predicts late aseptic failure of hip sockets," The Journal of Bone and Joint Surgery B, vol. 78, no. 3, pp. 422-426, 1996.

[8] J. M. Kabo, J. S. Gebhard, G. Loren, and H. C. Amstutz, "In vivo wear of polyethylene acetabular components," Journal of Bone and Joint Surgery B, vol. 75, no. 2, pp. 254-258, 1993.

[9] S. A. Sayeed, M. A. Mont, C. R. Costa et al., "Early outcomes of sequentially cross-linked thin polyethylene liners with large diameter femoral heads in total hip arthroplasty," Bulletin of the NYU Hospital for Joint Diseases, vol. 69, supplemet 1, pp. S90S94, 2011.

[10] K.-S. Park, J.-K. Seon, K.-B. Lee, and T.-R. Yoon, “Total hip arthroplasty using large-diameter metal-on-metal articulation in patients with neuromuscular weakness," The Journal of Arthroplasty, 2013.

[11] S.-J. Lim, K.-H. Lee, S.-H. Park, and Y.-S. Park, "Medium-term results of cementation of a highly cross-linked polyethylene liner into a well-fixed acetabular shell in revision hip arthroplasty," The Journal of Arthroplasty, 2013.

[12] J. M. Gililland, L. A. Anderson, J. Erickson, C. E. Pelt, and C. L. Peters, "Mean 5-year clinical and radiographic outcomes of cementless total hip arthroplasty in patients under the age of 30," BioMed Research International, vol. 2013, Article ID 649506, 7 pages, 2013.

[13] C. H. Lohmann, H. Meyer, J. V. Nuechtern et al., "Periprosthetic tissue metal content but not serum metal content predicts the type of tissue response in failed small-diameter metal-on-metal total hip arthroplasties," The Journal of Bone and Joint Surgery A, vol. 95, no. 17, pp. 1561-1568, 2013.

[14] K. H. Koh, Y.-W. Moon, S.-J. Lim, H. I. Lee, J. W. Shim, and Y.-S. Park, "Complete acetabular cup revision versus isolated liner exchange for polyethylene wear and osteolysis without loosening in cementless total hip arthroplasty," Archives of Orthopaedic and Trauma Surgery, vol. 131, no. 11, pp. 1591-1600, 2011.

[15] L. Olivecrona, H. Olivecrona, L. Weidenhielm, M. E. Noz, G. Q. Maguire Jr., and M. P. Zeleznik, "Model studies on acetabular component migration in total hip arthroplasty using CT and a semiautomated program for volume merging," Acta Radiologica, vol. 44, no. 4, pp. 419-429, 2003.

[16] H. Olivecrona, L. Weidenhielm, L. Olivecrona et al., "A new CT method for measuring cup orientation after total hip arthroplasty: a study of 10 patients," Acta Orthopaedica Scandinavica, vol. 75, no. 3, pp. 252-260, 2004.

[17] A. Jedenmalm, F. Nilsson, M. E. Noz et al., "Validation of a 3D CT method for measurement of linear wear of acetabular cups: a hip simulator study," Acta Orthopaedica, vol. 82, no. 1, pp. 3541, 2011.

[18] R. Gupta, M. Grasruck, C. Suess et al., "Ultra-high resolution flat-panel volume CT: fundamental principles, design architecture, and system characterization," European Radiology, vol. 16, no. 6, pp. 1191-1205, 2006.

[19] L. Olivecrona, J. Crafoord, H. Olivecrona et al., "Acetabular component migration in total hip arthroplasty using CT and a semiautomated program for volume merging," Acta Radiologica, vol. 43, no. 5, pp. 517-527, 2002.

[20] R. J. T. Gorniak, E. L. Kramer, G. Q. Maguire Jr., M. E. Noz, C. J. Schettino, and M. P. Zeleznik, "Evaluation of a Semiautomatic 3D Fusion Technique Applied to Molecular Imaging and MRI Brain/Frame Volume Data Sets," Journal of Medical Systems, vol. 27, no. 2, pp. 141-156, 2003.

[21] J. M. Bland and D. G. Altman, "Statistical methods for assessing agreement between two methods of clinical measurement," The Lancet, vol. 1, no. 8476, pp. 307-310, 1986.

[22] J. Ranstam, L. Ryd, and I. Onsten, "Erratum: Accurate accuracy assessment: review of basic principles," Acta Orthopaedica Scandinavica, vol. 71, no. 1, pp. 106-108, 2000.

[23] E. Vandenbussche, M. Saffarini, U. Hansen et al., "Measurement of femoral head penetration in polyethylene using a 3dimensional CT-scan technique," Acta Orthopaedica, vol. 81, no. 5, pp. 563-569, 2010.

[24] H. Malchau, J. Karrholm, Y. X. Wang, and P. Herberts, "Accuracy of migration analysis in hip arthroplasty: digitized and conventional radiography, compared to radiostereometry in 51 patients," Acta Orthopaedica Scandinavica, vol. 66, no. 5, pp. 418-424, 1995.

[25] J. M. Martell, E. Berkson, R. Berger, and J. Jacobs, "Comparison of two and three-dimensional computerized polyethylene wear analysis after total hip arthroplasty," Journal of Bone and Joint Surgery A, vol. 85, no. 6, pp. 1111-1117, 2003.

[26] T. Von Schewelov, L. Sanzén, N. Börlin, P. Markusson, and I. Önsten, "Accuracy of radiographic and radiostereometric wear measurement of different hip prostheses: an experimental study," Acta Orthopaedica Scandinavica, vol. 75, no. 6, pp. 691700, 2004.

[27] I. C. Clarke, K. Black, C. Rennie, and H. C. Amstutz, "Can wear in total hip arthroplasties be assessed from radiographs?" Clinical Orthopaedics and Related Research, vol. 121, pp. 126-142, 1976.

[28] C. J. Sychterz, C. A. Engh Jr., N. Shah, and C. A. Engh Sr., "Radiographic evaluation of penetration by the femoral head into the polyethylene liner over time," The Journal of Bone and Joint Surgery A, vol. 79, no. 7, pp. 1040-1046, 1997.

[29] P. A. Devane, J. G. Horne, K. Martin, G. Coldham, and B. Krause, "Three-dimensional polyethylene wear of a press-fit titanium prosthesis: Factors influencing generation of polyethylene debris," Journal of Arthroplasty, vol. 12, no. 3, pp. 256-266, 1997.

[30] J. M. Martell, J. J. Verner, and S. J. Incavo, "Clinical performance of a highly cross-linked polyethylene at two years in total hip 
arthroplasty: a randomized prospective trial," The Journal of Arthroplasty, vol. 18, no. 7, pp. 55-59, 2003.

[31] H. Baldursson, N. Egund, L. I. Hansson, and G. Selvik, "Instability and wear of total hip prostheses determined with roentgen stereophotogrammetry," Archives of Orthopaedic and Traumatic Surgery, vol. 95, no. 4, pp. 257-263, 1979.

[32] J. Kärrholm, P. Herberts, P. Hultmark, H. Malchau, B. Nivbrant, and J. Thanner, "Radiostereometry of hip prostheses: review of methodology and clinical results," Clinical Orthopaedics and Related Research, no. 344, pp. 94-110, 1997.

[33] L. Olivecrona, A. Jedenmalm, P. Aspelin et al., "Assessing wear of the acetabular cup using computed tomography: an ex vivo study," Acta Radiologica, vol. 46, no. 8, pp. 852-857, 2005.

[34] Yves Ligier, “OSIRIS," http://www.expasy.ch/www/UIN/.

[35] D. Goldvasser, M. E. Noz, G. Q. Maguire Jr, H. Olivecrona, C. Bradgon, and H. Malchau, "A new technique for measuring wear in total hip arthroplasty using computerized tomography," The Journal of Arthroplasty, vol. 27, no. 9, pp. 1636-1640, 2012. 


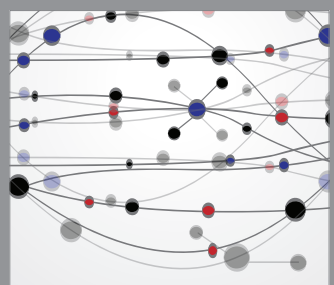

The Scientific World Journal
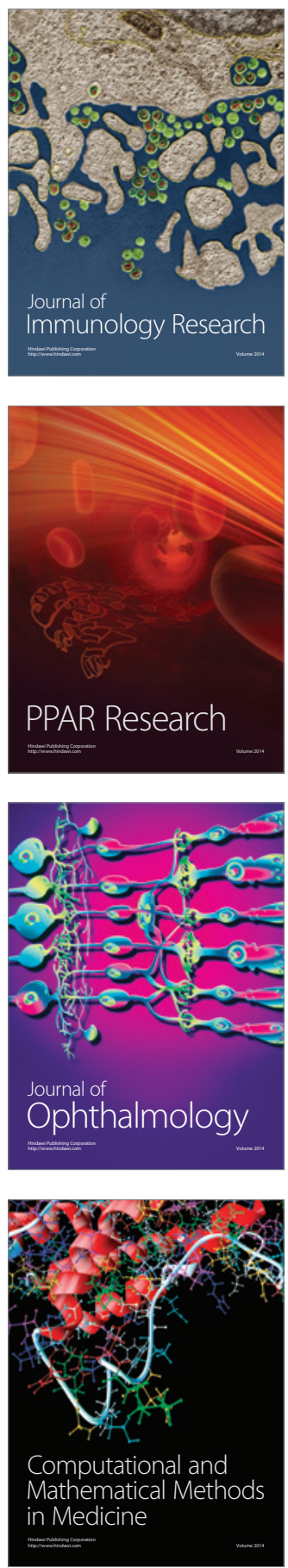

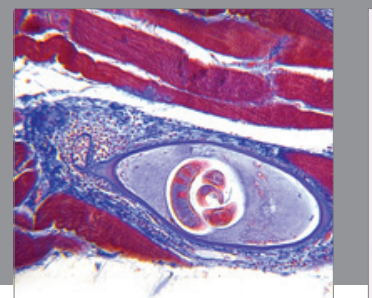

Gastroenterology

Research and Practice
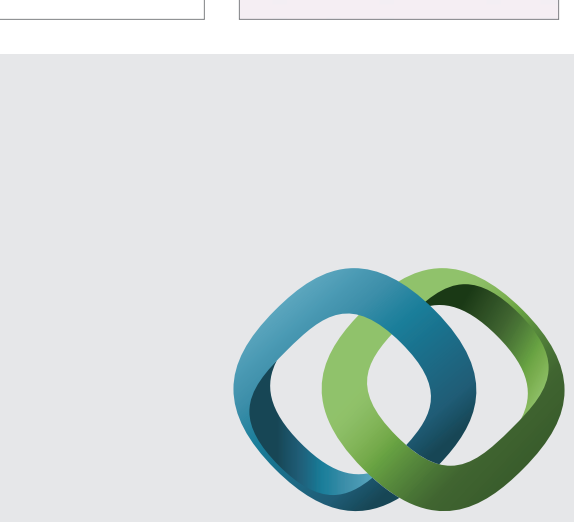

\section{Hindawi}

Submit your manuscripts at

http://www.hindawi.com
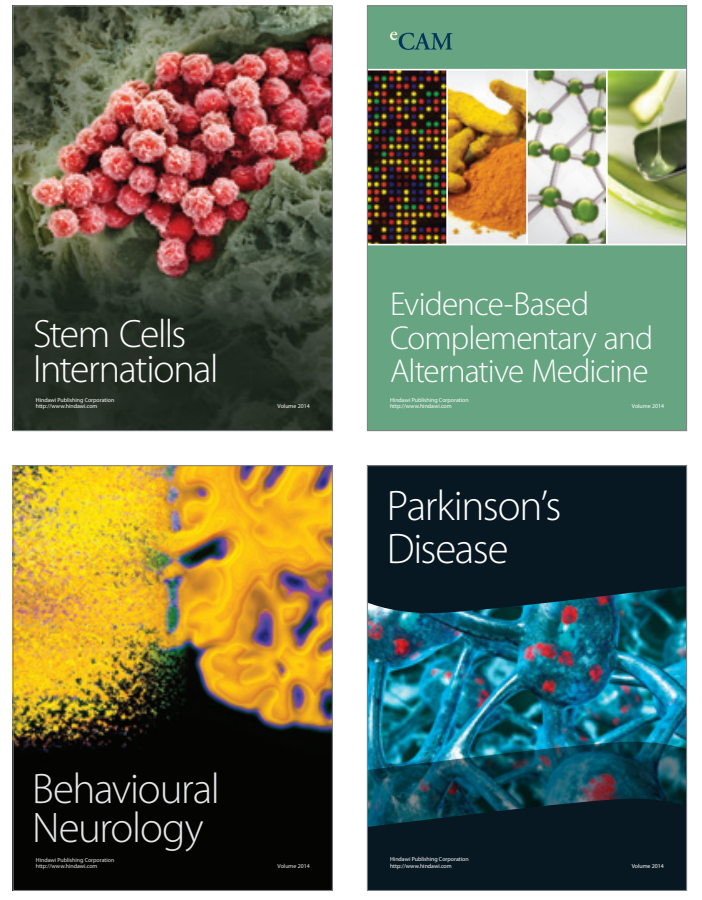
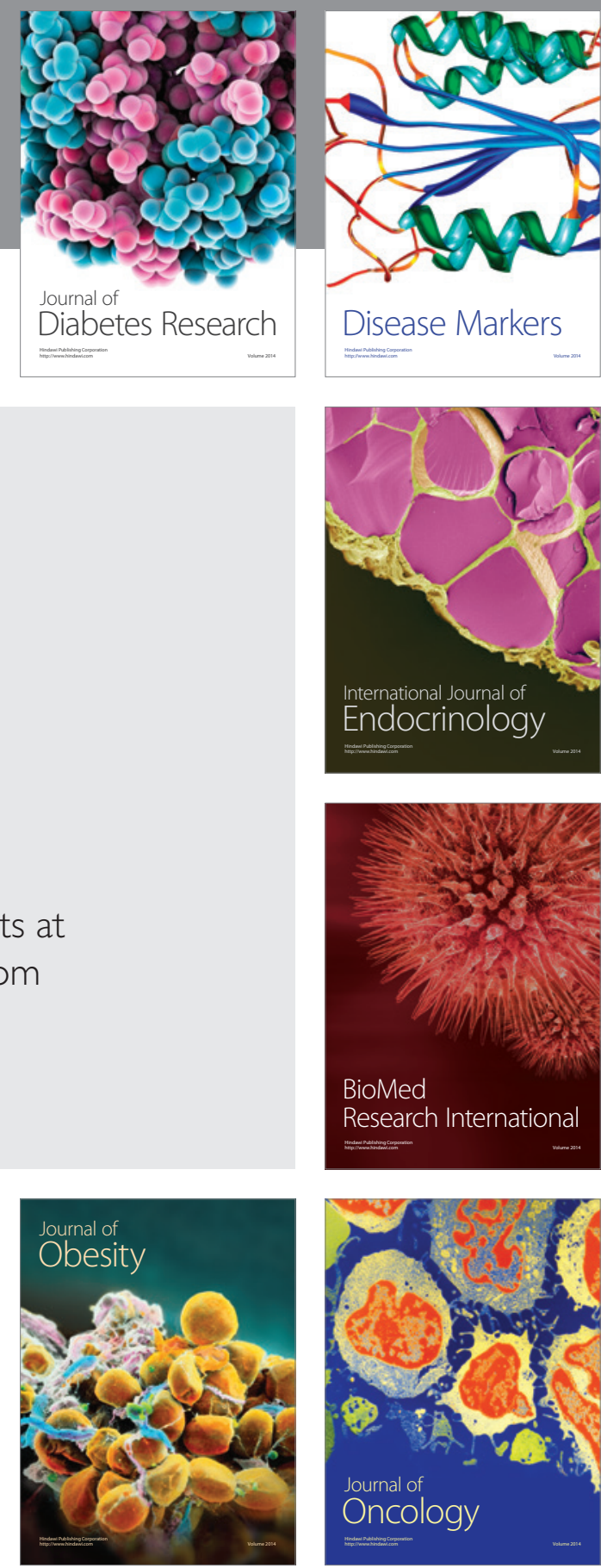

Disease Markers
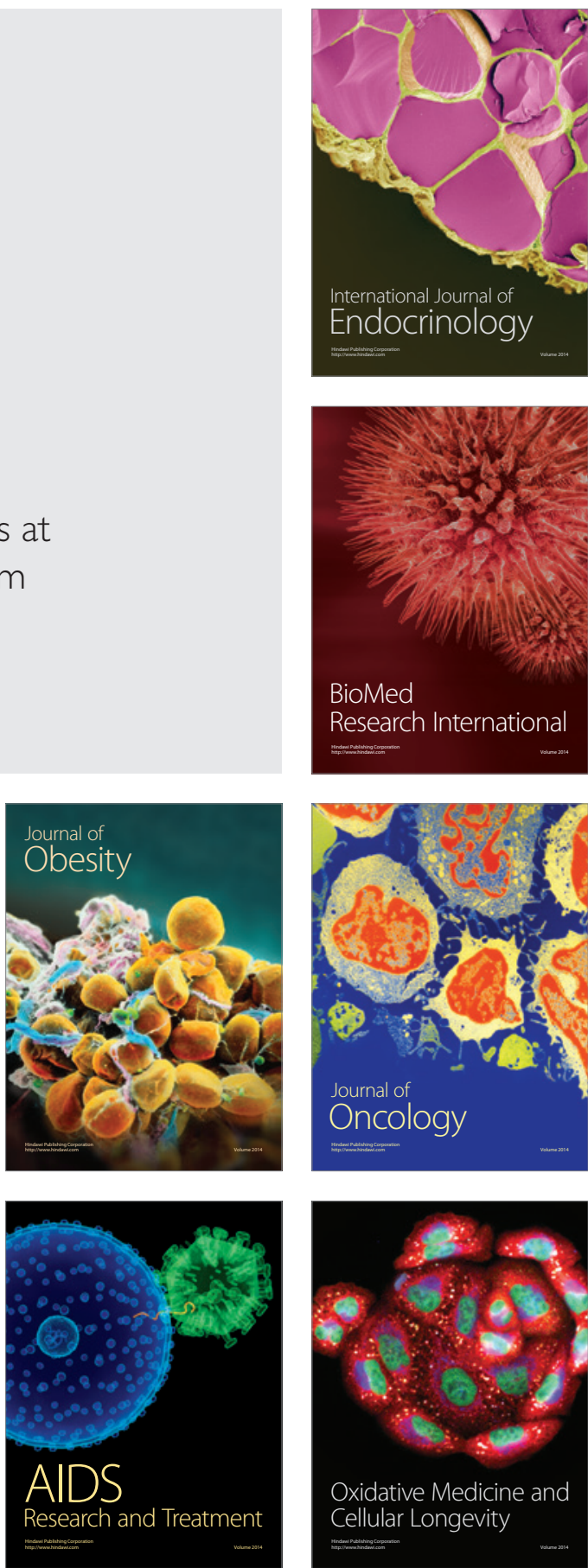